\title{
Is there a role for exercise in the prevention of osteoporotic fractures?
}

\author{
Olga M Rutherford
}

\begin{abstract}
Objectives-To examine whether there is a role for exercise in improving bone mineral density (BMD), particularly in postmenopausal women. The effects of different types of exercise are examined together with their effects at selected skeletal sites. The role of activity in reducing falls and hip fractures will also be considered as well as the potentially negative effects of excessive exercise.

Methods-A literature search over the past 20 years was conducted and landmark papers selected.

Results-Certain types of exercise have been found to exert moderate benefits on BMD of the wrist, spine, and hip. Most studies do not detect a difference between the effects of endurance activities and strength training for BMD of the spine. It has been more difficult to isolate the optimal type of activity for effecting an osteogenic response at the hip, but recent evidence suggests that high impact work such as stepping and jumping may be effective at this site. The combination of hormone replacement therapy and exercise would appear to be more effective than either intervention on its own. Certain types of exercises have additional benefits, such as muscle strengthening, which could reduce the incidence of falls. Excessive exercise can lead to menstrual disturbances in female athletes and this in turn can cause bone loss, particularly from the spine.

Conclusions-Exercise across the life span should be encouraged in order to maximise peak bone mass, reduce age related bone loss, and maintain muscle strength and balance. Although the effects of exercise on BMD later in life are small, epidemiological evidence suggests that being active can nearly halve the incidence of hip fractures in the older population. This effect is most probably multifactorial through the positive effects on bone, muscle strength, balance, and joint flexibility. Younger women should be aware of the dangers to the skeleton of menstrual disorders.
\end{abstract}

Keywords: exercise; osteoporosis; bone; fracture; hormone replacement therapy
One of the major demographic changes occurring in developed countries is an increase in the number of people over the age of 60 . With a shift to an increasingly elderly population, there is a growing need to maintain health and independence in older age. Within the older population, osteoporotic fractures, particularly of the hip, are a major health problem. ${ }^{1}$ Osteoporosis is a condition characterised by a low bone mass and poor bone architecture. Trabecular plates thin and develop holes and eventually whole plates can be lost. Cortical bone also thins, and these processes render bones fragile and prone to fracture after minimal trauma. In the United Kingdom alone the problem affects up to one in three women and one in 12 men. There is estimated to be an osteoporotic fracture every three minutes, and the associated morbidity and mortality is very high. The total cost to the NHS is in the region of $£ 940 \mathrm{~m}$ per year and is increasing by $10 \%$ per year. The major fracture sites are the vertebrae, hip, and radius. In men and women, fractures of the hip and spine rise exponentially with increasing age, particularly in women after the menopause. The incidence of wrist fractures starts earlier but levels off after about 60 years; this is most probably due to slower reaction times in the older person which prevents them from extending the arm to break a fall. The incidence of osteoporotic fractures is about double in women compared with men which is due in part to a generally lower bone density across the age span.

Many fractures arise because of a fall, and nearly one third of people over the age of 65 fall each year. Falls account for $82 \%$ of all accidental deaths in the home in the over $75 \mathrm{~s}^{2}$ and many of these deaths are due to fractures. There are many factors, other than bone strength that can predispose someone to falls and fractures. These include low muscle strength, poor balance and eyesight, stiff or painful joints, slow reaction times, poor nutrition, psychoactive drugs, and poor functional ability. ${ }^{3-6}$ Several of these could be improved with an appropriate exercise regimen. It is therefore the purpose of this review to look at the role of exercise both in maintaining bone density and reducing the risk of falls. The potential adverse effect to the skeleton of excessive exercise will also be considered. 


\section{Methodology}

The studies were selected from a Medline search over the past 20 years and from material already known to the author. Intervention studies had to meet the criteria that there was a suitably matched control group and that details of the exercises were given.

\section{Muscle strength in older age}

The importance of muscle weakness in older age cannot be underestimated. In a cross sectional study of healthy British women, quadriceps strength decreased on average by $40 \%$ between the third and eighth decade. ${ }^{7}$ In frail older people the loss may be greater. Part, but not all, of this weakness is due to muscle atrophy (sarcopenia). The quality of muscle also appears to be affected by age such that the normalised (or specific) force is also reduced. ${ }^{78}$ The decrease in specific force is particularly evident over the menopause and can be prevented with hormone replacement therapy (HRT). ${ }^{89}$ In women with osteoporosis, the specific force was found to be extremely low for their age. ${ }^{10}$ This suggests that the factors implicated in skeletal loss affect the ability of the muscle to generate force. The muscular forces exerted on bone are probably one of the key factors in maintaining bone health. Strategies aimed at preventing bone loss should also aim to strengthen muscle. Aside from the potential importance of muscle strength in fall risk, a good muscle mass may also be protective against fracture after a fall as the tissue can act as a cushion absorbing some of the impact forces.

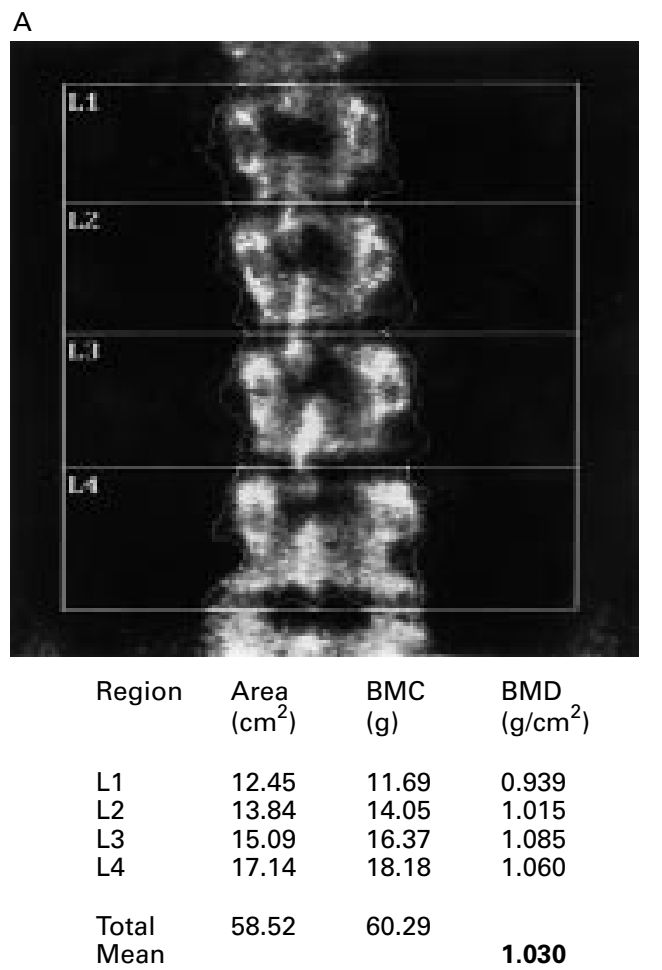

Measuring the problem

In the past $10-15$ years there has been an enormous increase in research into osteoporosis. This increase in attention is partly due to the advances in the technology for measuring bone density in vivo accurately, quickly, cheaply, and with a low radiation dose. Bone mass or density is an important measure as it is one of the major determinants of bone strength. The World Health Organisation recently issued guidelines for the diagnosis of osteoporosis or osteopenia (low bone mass) for the postmenopausal population who have not already sustained a fracture. Osteoporosis is defined as a bone mineral density (BMD) more than 2.5 $\mathrm{SD}$ below the mean for the young normal reference population, while osteopenia is a BMD between 1 and $2.5 \mathrm{SD}$ below the young mean. ${ }^{11}$

The most commonly used technique for measuring BMD is dual energy $x$ ray absorptiometry (DEXA). ${ }^{12}$ Although invaluable as a diagnostic and research tool, users need to be aware of some of the limitations of this technology. The scanner creates a frontal projection of the skeletal site of interest and calculates an areal density. At sites like the hip and radius, it is essential that the orientation of the bone is the same on each scanning occasion to ensure the same areal projection. When the lumbar vertebrae are scanned, caution needs to be taken if vertebrae are damaged, particularly if wedge or crush fractures, which may not be apparent on the frontal image, are present (fig 1). Damage or arthritic changes to the spine can artificially increase the calculated density and this is particularly common in older people. DEXA gives an integrated measure of

B

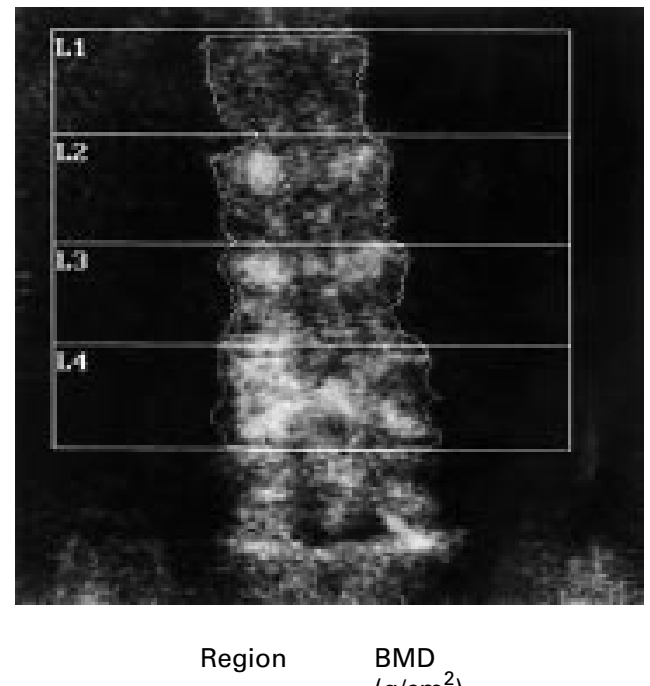

$\begin{array}{ll}\text { L1 } & 0.349 \\ \text { L2 } & 0.525 \\ \text { L3 } & 0.632 \\ \text { L4 } & 0.888 \\ & \\ \text { Mean } & \mathbf{0 . 6 2 8}\end{array}$

Figure 1 Dual energy x ray absorptiometry scan (Hologic QDR1000) of the lumbar vertebrae for (A) a healthy young woman and (B) a patient with osteoporotic crush fractures. Note the higher bone mineral density (BMD) values for L3 and L4 because of the focal areas of high density. BMC, bone mineral content. 
both trabecular and cortical bone, and it is the former that may be more informative. Dual energy computerised tomography (CT) does give a true volumetric density, and trabecular bone in the vertebrae can be isolated from the cortical shell. CT is, however, expensive, not widely available, and has a high radiation dose, and for these reasons is not commonly used for measurement of bone density. Any radiographic technique needs to have a very high accuracy and precision. Changes to bone occur slowly, and after one year of treatment the increases can be in the region of only $1-2 \%$. In the future, magnetic resonance imaging may provide a valuable tool for looking at the structure of bone which may be more informative about bone strength than density alone.

Other non-radiographic methods for monitoring the impact of treatment on bone include blood and urinary markers of bone turnover (for a review see Calvo $e t \mathrm{al}^{13}$ ). Current markers of bone formation include bone specific alkaline phosphatase and osteocalcin. Bone resorption can be assessed from breakdown products of type I collagen such as deoxypyridinoline and the telopeptides. In postmenopausal women, bone turnover, particularly resorption, is very high. These markers are used widely for monitoring antiresorptive treatment such as HRT and bisphosphonates but have been less commonly used in exercise intervention trials. As these markers are susceptible to circadian, menstrual, and seasonal rhythms, care needs to be taken when choosing sampling times.

\section{Exercise and fractures}

The evidence that exercise may have a role to play in reducing fractures comes from both cross sectional and longitudinal studies. Many studies have been carried out and it is beyond the scope of this review to include them all. Rather, key examples will be used to highlight the effectiveness of a particular type of exercise or effects at specific fracture sites. There are a number of excellent reviews on this subject in the literature. ${ }^{14-17}$ The term exercise covers many forms of activity. These can be broadly divided into endurance training, strength/ resistance training, high impact work, walking, and aerobics/keep fit classes. Much of the initial work aimed at improving BMD concentrated on the types of endurance activity that had been shown to be beneficial for cardiovascular health. We are increasingly becoming aware that the same type of exercise may not be optimal for bone health or reduction of falls. However, some of the basic principles of training still apply and these have been summarised by the American College of Sports Medicine. (a) Principle of specificity: only sites loaded by the exercise may respond as the effects are localised. (b) Principle of overload: the training stimulus must exceed the normal loading experienced by the skeleton in everyday activities and, as the bone responds, the stimulus must be increased progressively. (c) Principle of reversibility: any positive effects of training on BMD will only be maintained as long as the exercise is continued. (d) Principle of initial values: the most benefit is likely to be achieved in those with the lowest initial BMD. For this reason it is extremely important that the control and exercise groups are matched for BMD before intervention. (e) Principle of diminishing returns: individuals appear to have a biological/genetic ceiling that determines the extent of improvement. As training progresses, this ceiling may be reached, and gains in BMD will slow and eventually plateau. Many of the intervention studies are too short for this to be clearly observed.

\section{Cross sectional studies}

Some of the strongest evidence that exercise can increase BMD comes from studies on highly athletic groups. Comparisons have been made between the bone density of skeletal sites in different types of athletes and sedentary controls. Nilsson and Westlin ${ }^{18}$ measured femoral bone density of male athletes including weight lifters, throwers, runners, soccer players, swimmers, active non-athletes, and sedentary men. Bone density varied with the amount and extent of loading placed on the femur, with the greatest density occurring in the weight lifters and lowest in the sedentary group. This pattern was reflected in leg muscle strength. Similar studies have been carried out in women. Heinonen et $a l^{19}$ showed that bone density of the hip and spine exhibited a stepwise pattern in female athletes, with the largest in squash players followed by aerobics participants, speed skaters, and sedentary controls. The importance of impact loading was shown by Fehling et $a l^{20}$ who compared regional $\mathrm{BMD}$ in female athletes participating in impact loading sports (volleyball and gymnastics) with that in women participating in active loading sports (swimming) and sedentary controls. The impact loading groups had high BMDs at most skeletal sites whereas there were no significant differences at any site between the swimmers and controls (fig 2).

The criticism about such comparative studies is that the high bone density may simply reflect a genetically determined strong musculoskeletal system which favours the participation of these women in high level sports rather than the training itself leading to an

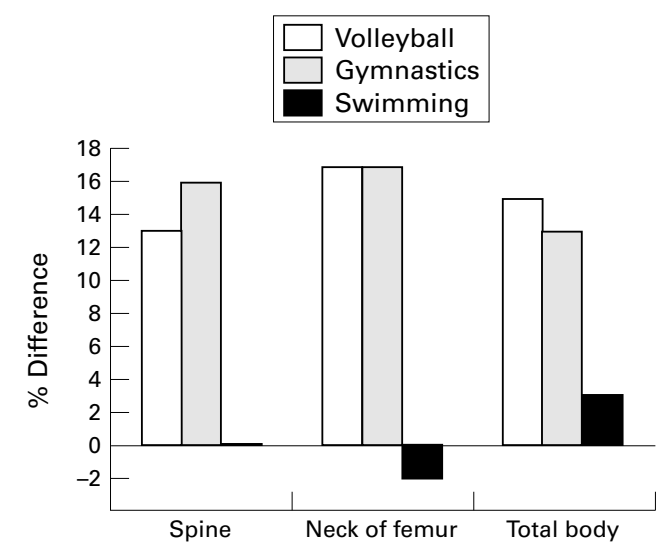

Figure 2 Percentage difference in bone mineral density of the spine, neck of femur, and total body in athletic women compared with sedentary controls. Redrawn from Fehling et al. ${ }^{20}$ 
increase in BMD. An argument against this comes from studies of asymmetric activities, such as tennis, where the playing arm has a larger bone mass than the non-playing arm. ${ }^{21}$ The differences observed in bone density between athletes and non-athletes can be large (10-15\%), and are often much greater than the changes measured as a result of prospective exercise intervention studies. There are several possible explanations for these observations: (a) high levels of activity early in life may be more beneficial than activity adopted later; $(b)$ the intervention studies are often of much shorter duration than the training history of the athletes; $(c)$ the athletes may have a genetic advantage in that their response to training is greater than that of many sedentary people. Not all athletic groups, however, show beneficial effects on bone and this will be discussed below.

\section{Exercise intervention}

In order to show conclusively that exercise can slow bone loss or cause bone accretion, it is necessary to perform longitudinal randomised intervention studies in the population of interest. Problems arise when trying to compare such studies as there are large variations in the type, intensity, and duration of the training. Additional confounding factors include wide differences in the age range of subjects, concurrent treatments, calcium supplementation, and skeletal sites investigated. Precise details of the exercises carried out are often scanty or missing. Despite these problems, we are beginning to understand more about the types of activities that can provide benefits for different skeletal sites. As yet we are not in a position to give definitive recommendations for the optimal type of exercise for the prevention of osteoporosis. Recently guidelines have been produced for physiotherapists dealing with patients either at risk of osteoporosis or with diagnosed osteoporosis, ${ }^{22}$ and the National Osteoporosis Society has produced a booklet giving advice on exercises that could help prevent osteoporosis. ${ }^{23}$ Both publications are based on the findings of research studies.

\section{Strengthening the wrist}

The Colle's fracture of the wrist is one of the first injuries to show an increase after the menopause. Because of its accessibility, the wrist is one of the sites that responds well to training, as long as the regimen is tailored to load the forearm. Squeezing a tennis ball for 30 seconds a day for six weeks showed significant benefits in the non-injured forearm of women who had already sustained a Colle's fracture. ${ }^{24}$ Alternatively resisted exercise involving twisting, compression, and bending resulted in increases averaging 3.8\%. ${ }^{25}$ More general keep fit and dance classes often fail to show benefits at the radius simply because the exercises were not tailored to stress that site.

\section{Increasing the bone density at the spine and hip}

More challenging to the researchers has been defining the optimal form of exercise that can have significant effects on bone density at the spine and hip. It has been difficult to isolate the type of exercise that places sufficient strain magnitude and of novel distribution to alter significantly bone turnover and remodelling, particularly in older age groups.

\section{WALKING}

One of the simplest and most accessible forms of exercise is walking. Unfortunately the available evidence suggests that this form of activity is insufficient to improve BMD at the spine or hip. It should be noted, however, that the subjects included in the studies have largely been fairly active, ambulant, and healthy. It has yet to be shown whether more inactive frail groups would show a more positive response. A one year study of treadmill walking at $70-85 \%$ of maximum heart rate together with calcium supplementation found no effect at the spine or forearm in postmenopausal women ${ }^{26}$ despite significant improvements in aerobic capacity. In recently menopausal women, walking did appear to attenuate the loss of bone at the spine when compared with controls. Nelson et $a l^{7}$ compared supervised walking and either moderate or high calcium diet on BMD of the spine and hip. The spine, but not the hip, showed a moderate improvement independent of calcium intake. A similar exercise regimen, but with no dietary supplementation, showed no effect on trabecular bone density in the spine. ${ }^{28}$ In a seven month trial, Hatori et a ${ }^{29}$ compared walking above (high intensity) or below (low intensity) the anaerobic threshold on spine BMD. The moderate intensity group showed a similar loss of bone to the controls, whereas the high intensity group showed a small improvement. Although the results are encouraging, it is unlikely that women would naturally adopt such an intense walking pace without continuous encouragement. Although walking does not appear to be particularly beneficial for bone health in the groups studied, it may have other benefits that could reduce the risk of fractures and should not be dismissed as an important activity for many other aspects of health.

LOW INTENSITY REPETITIVE EXERCISE

Many studies have investigated exercise regimens based on those recommended for training of the cardiovascular system; these have mainly involved some form of repetitive low force activity such as general keep fit classes. In one of the earliest studies, Krolner et $a l^{30}$ compared the effect of an eight month varied programme of walking, running, floor exercises, and ball games on the spine and forearm bone mineral content (BMC) with an age matched non-exercising control group. The exercise groups had a small $(3.5 \%)$, but significant, increase in BMC of the lumbar spine when compared with the decrease in the control group. No effect was seen at the forearm, probably because of a lack of loading at this site from this form of activity. The women were postmenopausal and had previ-

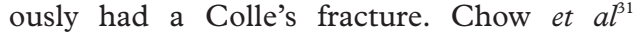
carried out a similar training programme but included an additional group in which light 
weights were attached to the wrists and ankles during the exercise classes. They measured total body calcium and this was improved in both exercise groups when compared with controls, but the group performing with the weights showed no additional benefit. The measurement technique was unable to detect any site specific effect.

In a more complex study design, Dalsky et $a l^{32}$ carried out a study in which the training included walking, running, and stair climbing in women aged 55-70. Calcium supplements were given at a dose of $1500 \mathrm{mg} /$ day regardless of dietary intake and subjects were randomly divided into exercise and non-exercise groups. In the first stage of the study, exercise was carried out for nine months. Some subjects in the exercise group then stopped training and the others continued to train for a further 13 months. After this second training phase, the exercise was stopped and subjects were followed up after a further 12 month detraining period. After the first study period the exercise group had a significant increase of $6 \%$ in spine BMC. After the second phase the exercise maintained this increase but did not improve it further. Those that had stopped training had returned to their baseline BMC. This study highlights many aspects outlined in the general principles for training discussed above. Firstly, within the exercise group some did not benefit from exercise and carried on losing bone while others had very large increases of up to $15 \%$. This could be due to different intensities of training, differences in initial BMC, or differences in genetic potential to respond to training. As with any intervention treatments, it highlights the need to monitor response and not assume that every person will respond positively. Secondly, it demonstrates the plateau effect in that the response was maintained but not increased in the second training phase. Thirdly, the results show that once exercise is stopped, the normal bone loss continues and the benefits are not maintained.

STRENGTH TRAINING

In the 1990s attention switched to the study of strength training. The rationale was based on findings from animal studies. ${ }^{33} 34$ These investigated in detail the type of mechanical strains that could maximise the osteogenic response. The greatest effects were seen when the magnitude and rate of strain was high, but required few repetitions, and when the strain was novel in magnitude or direction. The human equivalent of this type of strain exposure is strength, as opposed to endurance, training. Pruitt et $a l^{3536}$ conducted two studies on strength training, one in early postmenopausal women and one in women over 65 . In the first study on the younger women, there was a significant effect of training at the lumbar spine averaging $1.6 \%$, but no effect at the hip or forearm. In the second study on the older women, they compared high and low intensity strength training. Despite improvements in strength in both exercise groups, there was no effect on BMD at either the spine or hip for either group. Conversely Kerr et $a^{\beta^{7}}$ did find an effect of high intensity strength training on
BMD of several regions on the hip: the trochanter, intratrochanteric area, and Ward's triangle. They found no effect at the neck of femur and no effect of low intensity (endurance) strength training. Their population were all postmenopausal and ranged in age from 40 to 70 years. Both types of exercise improved muscle strength to a similar extent, and these improvements correlated with the bone changes at several sites in the high intensity group. Nelson $e t a p^{\beta 8}$ also found small, but significant, effects of a one year high intensity strength training regimen on BMD of the spine, hip, and total body in 50-70 year old women. In addition, there was a significant improvement in muscle strength, muscle mass, and balance, all of which are implicated in fall risk.

As with much of the literature in this field it is difficult to reconcile the different results from similar regimens in seemingly similar populations. The overall message, however, is that, at least for the spine, strength training can be effective but has no added benefit for BMD over the more endurance based regimens. The added benefit of increased muscle strength may, in the longer term, result in a greater effect on fracture risk. As yet this has not been assessed and most of the studies only last one year; a much longer follow up would be required to determine effects on the incidence of falls and fractures.

\section{HIGH IMPACT EXERCISE}

Many of the studies so far discussed have been ineffective at increasing BMD at the hip. As this is the most serious fracture site, it is essential that safe, affordable, and accessible exercise is defined. One of the first studies to be effective at this site was by Bassey and Ramsdale $^{39}$ in which they used jumping to impart high impact forces to the hip in premenopausal women. After six months there was a significant increase of $3.4 \%$ at the greater trochanter, but no other site at the hip and no change at the spine. Initially they modified the exercise before extending it to an older age group (50-60 years) and instead of jumping the women carried out heel drops. ${ }^{40}$ After one year no effect was seen at any skeletal site and the study was then repeated using jumps. ${ }^{41}$ Again, no effect was seen, so the older women were showing a different response to the younger group, which may be related to the oestrogen status of the subjects although those on HRT had a similar response to non-HRT users.

In the light of the initial encouraging findings of Bassey and Ramsdale ${ }^{39}$ in the premenopausal women, another study incorporated jumping, stepping, marching, and side stepping into an exercise class designed specifically for the over $50 \mathrm{~s}^{42}$ The study involved both postmenopausal women and men over 50 , none of whom were on calcium or HRT. After one year there were significant increases in hip, but not spine, BMD. The increases were in the range $1.6-2.2 \%$ depending on the site on the hip. Some of the subjects continued for a second year in which the spine BMD increased significantly and the changes in hip BMD were 
maintained. This type of exercise also completely reversed the age related loss of muscle strength, with the training group increasing quadriceps strength by $10 \%$ and the matched controls decreasing it by the same amount. Hip and shoulder flexibility also improved as a result of the training. Urinary excretion of pyridinoline and deoxypyridinoline were measured to assess the impact of the exercise on bone resorption. Both markers significantly decreased during the first six months of exercise and then returned to baseline values. This suggests that the exercise was suppressing osteoclastic bone resorption. Similar findings on the response to high impact work have been obtained in younger subjects. ${ }^{43}$ These studies lend support to the idea of high impact work being a good osteogenic stimulus at the hip. Extreme caution needs to be observed, however, when recommending this form of exercise for a frail population. High impact work would be contraindicated for those with impaired balance, osteoporosis/osteopenia, osteoarthritis in load bearing joints, or artificial joints. Even in relatively healthy older subjects, the exercises should only be introduced gradually into classes after an initial progressive skill specific training period to allow soft tissue adaptation and the requisite safe technique to be learnt.

\section{Exercise and HRT}

Many of the studies discussed have used calcium supplementation to bring subjects up to the recommended daily allowance. Less well studied has been the interaction between HRT and exercise. Several studies have shown that in the oestrogen replete sate, the response to training is greater. In the most comprehensive study to date, Kohrt et $a l^{44}$ compared four groups of women aged $60-72$ years. The first acted as a control, the second took HRT, the third exercised, and the fourth took HRT and exercised. Variables studied were bone density of the spine, hip, and total body, bone formation (osteocalcin), body composition, muscle strength, and estimated $\mathrm{VO}_{2} \mathrm{MAX}$. The exercise programme involved two months of flexibility exercises followed by nine months of walking, jogging, and stair climbing/ascending. Calcium intake was adjusted to about 1500 $\mathrm{mg} /$ day. After the initial 11 months, there was a six month follow up phase in which those on HRT remained on treatment. Women in exercise groups were encouraged to continue exercising but most reduced both the number of exercise sessions and intensity during this phase. Both the exercise and HRT alone brought about increases in BMD of the total body, spine, neck of femur, and Ward's triangle. HRT plus exercise increased BMD at all sites measured (spine, hip, total body) and was more effective than HRT alone in increasing BMD at the spine and total body and more effective than exercise alone at the total body, spine, and trochanter. Serum osteocalcin was reduced in both groups taking HRT but in no other group. HRT is known to be antiresorptive and reduce bone turnover. Both exercise groups had improvements in lean tissue mass and $\mathrm{VO}_{2} \mathrm{MAX}$ and reductions in fat mass; these effects were not seen in the other two groups. In the follow up period, those women taking HRT maintained or further increased BMD, the effect being greater in those who exercised. Some of the improvements were maintained in those in the exercise alone group. These results show that, in the oestrogen replete state, older women show a greater response to exercise. In addition, exercise had additional benefits which could help reduce the risk of falls. Further studies are required to investigate the interaction of exercise and other treatment options such as the bisphosphonates.

\section{Exercise and fall prevention}

Certain forms of exercise have been recognised as an integral part of multifactorial intervention programmes for the reduction of falls. Despite the recognition of the importance of exercise, there are no specific guidelines on falls management exercises for the older person. Many of the studies that have looked at the impact of exercise on falls have had inherent design faults. Many have relied on subject's recall to document the number of falls in the preceding year, and in an elderly population this may be very inaccurate. Others have included subjects with no history of falling, and it is therefore not surprising that a reduction in falls was not observed. Several trials have used exercise of insufficient intensity or duration to effect meaningful adaptations, and often the exercises are not targeted specifically at the factors that increase the risk of falls. Some of the key risk factors are poor gait, balance, muscle strength, and confidence. In a one year home based study, Campbell et $a l^{45}$ found a reduction of $20-30 \%$ in falls in women over 80 . Each subject was prescribed a particular set of home exercises by a physiotherapist and were regularly contacted by phone to monitor progress and maintain motivation. Another successful intervention was Tai $\mathrm{Chi}^{46}{ }^{46}$ which resulted in a halving of falls.

Recently a falls management exercise programme $^{47}$ was specifically designed to provide practitioners with a framework of specific tailored progressive exercise guidelines which could be adapted to suit older people with a wide range of abilities. The activities include three dimensional Tai Chi based movement patterns, targeted strengthening and stretching exercises, dynamic postural and gait training, and functional floor and standing activities to improve neuromuscular skill and confidence. In addition to supervised classes, the subjects are also encouraged to do home based exercise aided by an exercise booklet. The women are also taught how to get up from the floor after a fall and are encouraged to wear hip protector pads in the classes. The effectiveness of this regimen in reducing falls is currently being assessed in a group of frequent fallers. In addition to falls, the other measurement outcomes of the study include lower limb muscle strength and power, functional ability, reaction time, balance, and bone density. Examination of the baseline data has disclosed that there can be considerable lower limb asymmetry in strength and power in frequent fallers; this is particu- 
larly evident for the eccentric strength of the quadriceps. ${ }^{48}{ }^{49}$ In some the difference between legs can be as great as $50-60 \%$, and overall the frequent fallers have a reduced lower limb power compared with age matched non-fallers. All of these factors could be implicated in the higher incidence of falling in this group and could be improved by regular exercise.

\section{The downside of exercise}

An important determinant of future fracture risk is the bone mass accrued during the childhood and teenage years. Peak bone mass is reached in the second to fourth decade and it is important that this is maximised. One of the factors that is important in determining peak bone mass is physical activity. An active lifestyle should therefore be encouraged throughout the life span. It has been shown that the benefit to bone is much greater if exercise is begun before puberty rather than after it. $^{50}$ There are, however, younger people who may compromise the development of bone density by the adoption of extreme levels of exercise. With the increasing participation of women in endurance sports, a condition known as the female athlete triad has been recognised. The triad refers to the condition in which there is amenorrhoea, eating disorders, and bone loss. Menstrual disorders are very common in certain athletes, most notably gymnasts, dancers, runners, and triathletes. ${ }^{51}$ Intense physical training, particularly aerobic exercise, can disrupt normal ovarian function by inhibiting the production of gonadotropin releasing hormone by the hypothalamus. ${ }^{52}$ The mechanism by which this occurs is not known but a number of factors could combine to alter hypothalamic function. These include low body weight and fat mass, endocrine changes associated with chronic exercise such as raised levels of cortisol and endorphins, and inadequate energy intake. Many of the women who develop such disorders have a later than average puberty which may be associated with the adoption of intense exercise early in life..$^{53}$ This in turn may prevent the establishment of a strong hypothalamic-pituitary-ovarian axis leading to disturbances later. In this condition there is an alteration of the normal pattern of sex steroid production with a reduction in oestradiol and/or a shortening of the luteal phase of the cycle. Several studies have now shown that many amenorrhoeic athletes have reduced bone density of the spine compared with sedentary controls and fail to show an increased BMD at the hip and total body which is normally found in their eumenorrhoeic counterparts $^{55-57}$ (fig 3). It would appear that the normal osteogenic response to bone loading is compromised in the oestrogen deplete state.

Low oestrogen levels and bone loss are common to both the amenorrhoeic athlete and postmenopausal woman. In the latter, bone turnover is raised, particularly osteoclastic resorption, and it has been assumed that the same would be the case in the athletes. This was investigated by measuring serum and urinary markers of bone turnover in amenor-

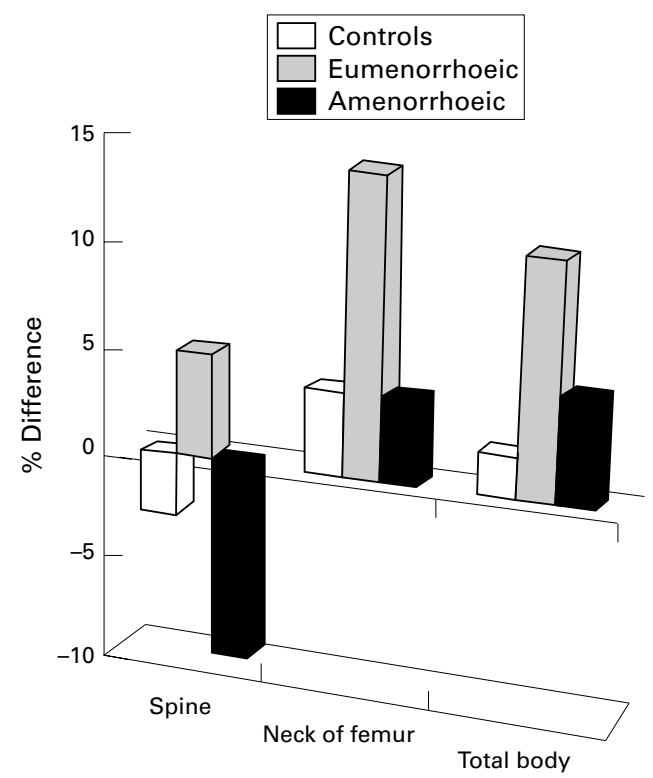

Figure 3 Percentage difference between bone mineral density of the spine, neck of femur, and total body for sedentary controls and athletic groups (eumenorrhoeic and amenorrhoeic) compared with predicted age matched data from the Lunar database. Redrawn from Stacey et $a l^{57}$ and the author's data.

rhoeic and eumenorrhoeic athletes (runners and triathletes) and sedentary controls. Neither the formation (osteocalcin and bone specific alkaline phosphatase) nor resorption (deoxypyridinoline) markers were increased in the amenorrhoeic group. ${ }^{57}$ The physiological effects of oestrogen and mechanical stress on bone turnover are thought, at least in part, to be exerted by an elevation in nitric oxide (NO) synthesis. Postmenopausal women have reduced NO levels, and both short and long term HRT elevate these. ${ }^{58} 59$ Administration of NO donors to oestrogen depleted rats prevents osteoporotic bone loss, ${ }^{60}$ and inhibition of $\mathrm{NO}$ synthesis suppresses the bone conserving action of oestradiol replacement. It was hypothesised that NO levels may also be low in amenorrhoeic athletes. The 24 hour excreted metabolites of NO were significantly reduced in this group despite having a significantly higher dietary nitrate intake. ${ }^{57}$ The amenorrhoeic athletes therefore resemble postmenopausal women in having reduced NO levels and spinal osteopenia but do not have raised bone turnover. The mechanism of bone loss in this group still remains to be clarified.

Less attention has been paid to elite male athletes. A pilot study found that male triathletes did not have increased bone density at the spine or total body compared with sedentary controls despite their high levels of activity, and the testosterone levels were significantly lower. ${ }^{61}$ Effects similar to those seen in the women could be occurring to the male hypothalamic-pituitarygonadal axis leading to a reduced production of testosterone.

Injuries to the musculoskeletal system are much more common in amenorrhoeic athletes, in particular the development of stress fractures. ${ }^{62}$ There has even been a report of an osteoporotic fracture occurring in a young female athlete. ${ }^{63}$ It is important that the athletes 
and those responsible for their care are aware of the potential risks to the skeleton of extended periods of menstrual irregularities, as it remains to be shown whether these skeletal deficits can be reversed by the resumption of menses or hormone replacement. ${ }^{64}$

\section{Conclusions}

The evidence is growing that some forms of exercise are able to reduce or reverse the age related loss of bone. Often the effects appear small but epidemiological data indicate that a history of physical activity can reduce the incidence of fractures, particularly of the hip, by up to half. ${ }^{65}{ }^{66}$ Weight bearing activity appears to be particularly effective, including walking and stair climbing. This effect is probably multifactorial in nature through improved $\mathrm{BMD}$, muscle strength, and balance. As yet we do not know the optimum or minimum form of exercise to achieve this reduction. Many of the studies have been carried out in relatively healthy subjects, and safe exercise regimens for people who have already sustained a fracture need to be defined. As with any intervention aimed at reducing fractures, exercise should be implemented as early as possible before trabecular plates have developed holes or been lost. Once this stage has been reached, no treatment is able to replace the plates and the bone strength will be permanently compromised.

I would like to thank Susie Dinan for her invaluable help and advice on both the preparation of this review and in the design and implementation of research studies.

1 Kannus P, Niemi S, Palvanen M, et al. Fall-induced injuries among elderly people. Lancet 1997;350:1174

2 Wallis R. Epidemiological overview of the health of elderly people. London: Department of Health, 1991

3 Lord SR, McLean D, Strathers G. Physiological factors associated with injurious falls in older people living in the community Gerontology 1992:38:338-46.

4 Lord SR, Sambrook PN, Gilbert C, et al. Postural stability, falls and fractures in the elderly. Results from the Dubbo osteoporosis epidemiology study. Med f Aust 1994;160: 684-5.

5 Salgado R, Lord SR, Packer J, et al. Factors associated with falling in elderly hospital patients. Gerontology 1994;40: falling in

6 Duncan PW, Studenski S, Chandler J, et al. Functional reach: predictive validity in a sample of elderly male veterans. $\mathcal{F}$ Gerontol 1992;47:M93-8.

7 Rutherford OM, Jones, DA. The relationship of muscle and bone loss and activity levels with age in women. Age Ageing 1992;21:286-93.

8 Phillips SK, Rook K, Siddle NC, et al. Muscle weakness in women occurs at an earlier age than in men, but strength is preserved by hormone replacement therapy. Clin Sci 1993; 84:95-8.

9 Skelton DA, Phillips SK, Bruce SA, et al. Hormone replacement therapy increases isometric muscle strength of adductor pollicis in post-menopausal women. Clin Sci 1999;96:357-64.

10 Jones DA, Rutherford OM. Effect of ageing and osteoporosis on the force-generating capacity of the quadriceps mussis on the force-generating capacity of the qua
cle in women. F Physiol (Lond) 1990;423:84P.

11 The WHO study group. Assessment of fracture risk and its application to screening for postmenopausal osteoporosis. Geneva: World Health Organisation, 1994;5-6.

12 Wahner HW, Fogelman I. The evaluation of osteoporosis: dual energy $X$-ray absorptiometry in clinical practice. London: Martin Dunitz, 1994

13 Calvo MS, Eyre DR, Gundberg CM. Molecular basis and clinical application of biological markers of bone turnover. Endocr Rev 1996;17:333-60.

14 Ernst E. Exercise for female osteoporosis. A systematic review of randomised clinical trials. Sports Med 1998;25:359-63.

15 Gutin B, Kasper MJ. Can vigorous exercise play a role in osteoporosis prevention? A Review. Osteoporos Int 1992:2:55-69.

16 Drinkwater BL. Does physical activity play a role in preventDrinkwater BL. Does physical activity play a role in preve
ing osteoporosis? Res $Q$ Exerc Sport 1994;65:197-206.

17 Forwood MR, Burr DB. Physical activity and bone mass: exercise in futility? Bone and Mineral 1993;21:89-112.

18 Nilsson BE, Westlin NE. Bone density in athletes. Clin Orthop 1971;77:179-82.
19 Heinonen A, Oja P, Kannus P, et al. Bone mineral density of female athletes in different sports. Bone and Mineral 1993; 23:1-14.

20 Fehling PC, Alekel L, Clasey J, et al. A comparison of bone mineral densities among female athletes in impact loading and active loading sports. Bone 1995;17:205-10.

21 Jones H, Priest JD, Hayes WC, et al. Humeral hypertrophy in response to exercise. F Bone foint Surg 1977;59:204-8.

22 Mitchell S, Creed G. Physiotherapy guidelines for the management of osteoporosis. Glasgow: Royal Infirmary, 1999.

23 National Osteoporosis Society. Exercise and bone health. Bath, NOS, 1999

24 Beverly MC, Rider TA, Evans MJ, et al. Local bone mineral response to brief exercise that stresses the skeleton. $B M \mathcal{F}$ 1989;299:233-5.

25 Ayalon J, Simkin A, Leichter I, et al. Dynamic bone loading exercises for postmenopausal women: effect on the density

26 Martin D, Notelovitz M. Effects of aerobic training on bone mineral density of postmenopausal women. $\mathcal{F}$ Bone foint Res 1993;8:931-6

27 Nelson ME, Fisher EC, Dilmanian FA, et al. A 1-y walking program and increased dietary calcium in postmenopausal women: effects on bone. Am f Clin Nutr 1991;53:1304-11.

28 Cavanaugh DJ, Cann CE. Brisk walking does not stop bone loss in postmenopausal women. Bone 1988;9:201-4.

29 Hatori M, Hasegawa A, Adachi $\mathrm{H}$, et al. The effects of walking at the anaerobic threshold level on vertebral bone loss in
postmenopausal women. Calcif Tissue Int 1993;52:411-14.

30 Krolner B, Toft B, Pors Nielson S, et al. Physical exercise as prophylaxis against involutional verte

31 Chow $R$, Harrison JE, Notarius $C$. Effect of two randomised exercise programmes on bone mass of healthy postmenoexercise programmes on bone mass of
pausal women. BMf 1987;295:1441-4.

pausal women. BMF 1987;295:1441-4.
Dalsky GP, Stocke KS, Ehsani AA, et al. Weight-bearing exercise training and lumber bone mineral content in postmenopausal women. Ann Int Med 1988;108:824-8.

33 Rubin CT, Lanyon LE. Regulation of bone formation by applied dynamic loads. F Bone foint Surg 1984;66:397-417.

34 Rubin CT, Lanyon LE. Regulation of bone mass by mechanical strain magnitude. Calcif Tissue Int 1985;37: 411-17.

35 Pruitt LA, Jackson RD, Bartels RL, et al. Weight-training effects on bone mineral density in early postmenopausa women. F Bone Miner Res 1992;7:179-85.

36 Pruitt LA, Taafe DR, Marcus R. Effects of a one-year highintensity resistance training program on bone mineral density in older women. $\mathcal{F}$ Bone Miner Res 1995;10:1788-95.

37 Kerr D, Morton A, Dick I, et al. Exercise effects on bone mass are site-specific and load-dependent. $\mathcal{F}$ Bone Miner Res 1996;11:218-25.

38 Nelson ME, Fiatarone MA, Morganti CM, et al. Effects of high intensity strength training on multiple risk factors for osteoporotic fractures. $¥ A M A$ 1994;272:1909-14.

39 Bassey EJ, Ramsdale SJ. Increase in femoral bone density in young women following high impact exercise. Osteoporos Int 1994;4:72-5.

40 Bassey EJ, Ramsdale SJ. Weight-bearing exercise and ground reaction forces: a 12-month randomized controlled trial of effects on bone mineral density in healthy postmenopausal women. Bone 1995;16:469-76.

41 Bassey EJ, Rothwell MC, Litlewood JJ, et al. Pre- and postmenopausal women have different bone mineral density responses to the same high impact exercise. $\mathcal{F}$ Bone Miner Res 1998;13:1805-13.

42 Welsh L, Rutherford OM. Hip bone mineral density is improved by high-impact exercise in post-menopausal women and men over 50 years. Eur 7 Appl Physiol 1996;74: women and 17 .

43 Heinonen A, Kannus P, Siavann H, et al. Randomised control trial of effect of high-impact exercise on selected risk factors for osteoporotic fractures. Lancet 1996;348:1343-7.

44 Kohrt W, Snead D, Slatopolsky E, et al. Additive effects of weight-bearing exercise and estrogen on bone mineral density in older women. $\mathcal{F}$ Bone Miner Res 1995;10:1303-11.

45 Campbell AJ, Robertson MC, Gardner MM, et al. Randomized controlled trial of a general practice programme of home based exercise to prevent falls in elderly women. BMF 1997;315:1065-9.

46 Wolf SL, Barnhart HX, Kutner NG, et al. Reducing frailty and falls in older persons: an investigation of tai chi and computerised balance training. F Am Geriatr Soc 1996;44: 489-97.

47 Skelton DA, Dinan SM. Exercise for falls management: rationale for an exercise programme aimed at reducing postural instability. Physiotherapy, Theory and Practice 1999; 15:105-20.

48 Kennedy J, Skelton DA, Rutherford OM. Lower limb muscle strength in frequently falling community dwelling women aged 65 and over. EGREPA Conference, France, 1999.

49 Skelton DA, Kennedy J, Rutherford OM. Lower limb muscle power in frequently falling community dwelling women aged 65 and over. Fournal of Aging and Physical Activity, in press.

50 Kannus P, Haapasalo H, Sankelo M, et al. Effect of starting age of physical activity on bone mass in the dominant arm of tennis and squash players. Ann Intern Med 1995;123:27-31.

51 Carbon RJ. Exercise, amenorrhoea and the skeleton. Br Med Bull 1992;48:546-60.

52 Loucks AB, Vaitukaitis J, Cameron JL, et al. The reproductive system and exercise in women. Med Sci Sports Exerc 1992;24:S288-93.

53 Malina RM. Menarche in athletes: a synthesis and hypothesis. Ann Hum Biol 1983;10:1-24. 
54 Stager JM, Hatler LK. Menarche in athletes: the influence of genetics and prepubertal training. Med Sci Sports Exerc

55 Drinkwater BL, Nilson $\mathrm{K}$, Chesnut $\mathrm{CH}$, et al. Bone mineral content of amenorrhoeic and eumenorrhoeic athletes. $N$ Engl f Med 1984;5:277-81.

56 Rutherford OM. Spine and total body bone mineral density in amenorrhoeic athletes. F Appl Physiol 1993;74:2904-8.

57 Stacey E, Korkia P, Hukkanen MVJ, et al. Decreased nitric oxide levels and bone turnover in amenorrhoeic athletes with spinal osteopenia. f Clin Endocrinol Metab 1998;83: 3056-61.

58 Cicinelli E, Ignarro LJ, Lograno $\mathrm{M}$, et al. Acute effects of transdermal estradiol administration on plasma levels of nitric oxide in postmenopausal women. Fertil Steril 1997;67:63-6.

59 Rosselli M, Imthurn B, Keller PJ, et al. Circulating nitric oxide (nitrite/nitrate) levels in postmenopausal women oxide (nitrite/nitrate) levels in postmenopausal women a two-year follow-up study. Hypertension 1995;25:848-53.
60 Wimalawansa SJ, De Marco G, Gangula P, et al. Nitric oxide donor alleviates ovariectomy-induced bone loss. Bone 1996;18:301-4

61 Smith R, Rutherford OM. Spine and total body bone mineral density and testosterone levels in male athletes. Eur $\mathcal{f}$ Appl Physiol 1993;67:330-4.

62 Bennell KL, Malcolm SA, Wark JD, et al. Skeletal effects of menstrual disturbances in athletes. Scand F Med Sci Sports 1997;7:261-73.

63 Wilson JH, Wolman RL. Osteoporosis and fracture complications in an amenorrhoeic athlete. $\mathrm{Br} f$ Rheumatol 1994;33:480-1

64 Keen AD, Drinkwater BL. Irreversible bone loss in former amenorrhoeic athletes. Osteoporos Int 1997;7:311-15.

65 Law MR, Wald NJ, Meade TW. Strategies for prevention of osteoporosis and hip fractures. BMF 1991;303:453-9.

66 Joakimsen RM, Magnus JH, Fonnebo V, et al. Physical activity and predisposition for hip fractures: a review. Osteoporos Int 1997;7:503-13.

\section{The GP training all the time even really slowly (TATT-ERS) runners}

Most of us have given up making New Years resolutions. However, this year, one person's resolution to run the Bristol half marathon has spread to many. In the inaugural run, there was a small core of hares in our pack but most of us broke into a sweat after 100 metres. An unforeseen benefit of the running pack is the conversation, with a mixture of cocktail party tattle, primary care group business, and straight continuing medical education, interspersed with stretches of quiet on long uphill grinds. Initially $40 \%$ of the session was spent running and talking followed by $60 \%$ talking and drinking.

We take our drugs after the event. The balance is shifting in favour of the exercise as deadlines and our first competitive event, the Long Ashton $10 \mathrm{~km}$ road race, approach. So far, no major life or relationship threatening disasters!

Competing against the clock has made us wiser: the sensible carbohydrate loading pasta meal is now taken the evening, rather than the hour, before the race. A handicap, not quite, lets just say we award ourselves a bonus for good behaviour. Hence, our true time is adjusted using a handicap formula calculated using a complex dataset that includes the runner's age, number of children, post-run beer consumption, pre-run pasta loading, partner support, BMI, cost of running shoes, kit, and accessories, and the ability to know how to adjust the pace of running in relation to things like pulse rate and hills. The exact formula is still in development; we are confident that the handicap times should enable us all to adjust our actual times in the half marathon to the 45 minutes that we all could do in ideal circumstances.

As dedicated self directed life long learners, we are absorbing all the jargon, uniform, and technology that goes with being a modern running. After the jargon comes the injuries, and the saying "There are 2 types of runners, the injured and the seriously injured" translates into our jogger's nipple, chapped thighs, and anterior knee pains in rotation. We have started our final altitude (and attitude) training and we will let you know how many of us make it to the finishing line in one piece.

MATT HOGHTON TERRY KEMPLE Brockway Medical Centre, Bristol 